\title{
Advanced Oncology Certified Nurse
}

National Cancer Institute

\section{Source}

National Cancer Institute. Advanced Oncology Certified Nurse. NCI Thesaurus. Code C71293.

A registered nurse who has worked at least 12 months out of the last 3 years as an oncology nurse. The nurse must also have worked at least 1,000 hours out of the last 2-

$1 / 2$ years in the field of oncology. 